\title{
Planificação de programas intergeracionais: Ilustrações com base numa freguesia da cidade do Porto
}

\section{Planning of intergenerational programs: Illustrations based in a parish of the city of Oporto}

\author{
Susana Villas-Boas*, Albertina Lima de Oliveira*, Natália Ramos**, Inmaculada Montero*** \\ * Universidade de Coimbra, ** Universidade Aberta de Lisboa, *** Universidade de Granada, Espanha
}

\begin{abstract}
Resumo
No presente trabalho apresentam-se os resultados do estudo sobre a análise de necessidades e potencialidades para a elaboração de programas educativos intergeracionais na freguesia do Bonfim da cidade do Porto. Começamos por apresentar dados do levantamento do perfil comunitário da referida freguesia, relacionados com as suas principais problemáticas, que resulta de três diferentes tipos de pesquisa: i) a pesquisa documental do que se sabe sobre a freguesia, ii) a pesquisa quantitativa, sobre as potencialidades e necessidades da população para o desenvolvimento de programas educativos intergeracionais e iii) a pesquisa qualitativa sobre as relações intergeracionais e os estereótipos e atitudes negativas de uma geração em relação às outras. E termina-se com a apresentação de programas educativos intergeracionais, desenhados a partir do cruzamento dos dados provenientes das três vias de investigação supramencionadas.
\end{abstract}

\begin{abstract}
This paper presents the results of the study on the analysis of needs and potentials for intergenerational educational programs in the parish of Bonfim from the city of Oporto. We begin by presenting data about the profile of the community that has been investigated in order to understand its main potentials and problems. Three different research paths were used in the investigation: i) documental research, ii) quantitative research about potentialities for the development of intergenerational educational programs and iii) qualitative research about intergenerational relations and gender stereotypes and also negative attitudes from one generation to the others. The paper ends with the presentation of intergenerational educational programs for the parish of Bonfim drawn from the intersection of the data from the three types of research mentioned.
\end{abstract}

\section{Introdução}

As pessoas, hoje, vivem mais tempo; pela primeira vez na história da humanidade coexistem no mesmo espaço temporal cinco a seis gerações (crianças, jovens, adultos jovens, adultos de meia-idade, adultos idosos, adultos de idade muito avançada). Porém esta coexistência não se traduz num maior contacto e diálogo entre pessoas de diferentes gerações, pelo contrário, as gerações estão cada vez mais separadas. As razões que levam a esta separação são múltiplas: as mudanças na estrutura familiar, as mudanças dos papéis sociais e de género, as mudanças na economia e no trabalho, o avanço tecnológico, as políticas e respostas sociais direcionadas a grupos-alvo específicos, a educação formal e não-formal que utiliza como critério de frequência a idade, entre outras. Estes fatores dificultam a organização de espaços físicos compartilhados, propícios à interação, construção de relações e transmissão de conhecimentos e aprendizagens entre as gerações mais novas e as mais velhas.

Sendo a educação intergeracional (EI) condição sine qua non para a existência da humanidade, porque o ser humano nasce, geneticamente, na condição de inacabado, tornando-se humano através do processo de socialização, as aprendizagens por meio do contacto e interação com os seus semelhantes humanos de outras gerações são vitais. É da estimulação e transmissão deliberada da cultura, de valores, atitudes, conhecimentos, técnicas, recordações, etc., que resulta a nossa humanidade (Savater, 2006). Torna-se, assim, necessário, nas sociedades contemporâneas, reforçar as oportunidades de contacto entre gerações e promover a educação intergeracional, atendendo ao crescente isolamento e separação a que têm vindo a ser votadas. Assim, quando, hoje, acentuamos a importância da EI, não nos referimos à educação que acontece espontaneamente, mas sim a uma educação sistemática, fruto de uma intenção, investigação e planeamento cuidadosos.

\section{A EI e os programas educativos Intergeracionais (PEI).}

A EI é um conceito recente, complexo, sem uma definição consensual e ainda no início do seu desenvolvimento. Todavia, da literatura é possível salientar alguns aspetos fundamentais relativos ao seu significado:

1) a EI é uma educação prática, focada no indivíduo que aprende e na aprendizagem. "O objetivo é mudar e transformar-se na aprendizagem com o outro" (Sáez, 2002, p. 104); 
2) a EI através do intercâmbio educacional entre as diferentes gerações promove o enriquecimento mútuo e o florescimento de comunidades, lugares e locais, e, em última instância, das nossas sociedades (Cabanillas, 2011; Mannion, 2012);

3) a EI conecta gerações e promove relações intergeracionais através da transmissão de conhecimento entre as gerações, seja das mais novas para as mais velhas ou vice-versa (Mannion, 2012);

4) a EI fundamenta-se na perspetiva da educação ao longo da vida, o que significa que a educação coexiste com o próprio tempo de vida, desde o berço até à morte, durante os primeiros anos de educação, a vida profissional, durante a adultez, em que a educação de adultos inclui todas as esferas da vida e na vida adulta tardia (Boström, 2014; Cabanillas, 2011; Brink, 2017).

5) A EI abrange todos os tipos de ensino e aprendizagem, o que significa que se desenvolve em variados contextos, tais como na família, na comunidade, na educação de adultos, no trabalho (Formosa, Karsovec \& Schmidt-Hertha, 2014) nas escolas e universidades, nos centros de dia, nas associações (Kaplan, 2001), em espaços livres e públicos (ONeil, 2016), etc.

6) A EI é uma ferramenta válida para responder a uma sociedade em mudança: otimiza as possibilidades de envelhecimento ativo, tem impacto na integração das pessoas de todas as idades que migram na busca de novas oportunidades profissionais; na redução de estereótipos de género e nas atitudes discriminatórias em função do sexo; no contexto das desigualdades sociais, através da interajuda; e na procura da melhoria pessoal dos indivíduos, etc. (Cabanillas, 2011; Ohsako, 2002; Seedsman; 2014).

7) A EI contribui para aumentar o conhecimento dos indivíduos sobre os mais variados temas de interesse; para a educação cívica, a educação ambiental, a educação familiar, a educação para a saúde; melhorar as relações intergeracionais; gerar respeito mútuo entre gerações; aumentar a reciprocidade e a cooperação entre as gerações; aumentar a participação e implicação dos indivíduos na comunidade e sociedade, o capital social e a coesão social; prevenir o declínio cognitivo durante o envelhecimento; aumentar o bem-estar e a qualidade de vida dos indivíduos; estabelecer redes de apoio social e facilitar a integração social; entre outras.

Assim, a EI é entendida como uma medida que reforça as relações sociais e intergeracionais, que através do intercâmbio de conhecimento, informações, pensamentos, ideias, sentimentos, experiências, habilidades, atitudes, etc., facilita a inovação de conhecimentos, de ideias, a transformação do indivíduo, e que através da cooperação (entre) e da participação das diferentes gerações contribui, igualmente, para o enriquecimento da comunidade.

A EI é colocada em prática, sobretudo, através dos programas intergeracionais que definimos como:
Um sistema, uma abordagem e uma prática em que todas as gerações, independentemente da idade, etnia, localização e estatuto socioeconómico, se unem no processo de gerar, promover e utilizar ideias, conhecimentos, habilidades, atitudes e valores de forma interativa com o objetivo de fomentar a melhoria pessoal e o desenvolvimento da comunidade. (Hatton-Yeo, 2002, p. 19)

Estes programas têm vindo a demonstrar que são um bom método para a intervenção social e educativa (Dorfman et al., 2002; Simons \& Cleary, 2006; Teater, 2016). Contudo, os especialistas chamam a atenção para a complexidade do ato de planificar e implementar, o qual exige tempo, conhecimento e muito cuidado. Como principais obstáculos, a literatura reporta: conseguir alcançar e selecionar participantes; conseguir que as pessoas de diferentes gerações aceitem encontrar-se, uma vez que os grupos geracionais têm preferências, necessidades e motivações diferentes que podem dificultar a participação; conseguir financiamento e recursos adequados para fazer um bom trabalho; dificuldades na organização de uma rede de instituições apropriadas; emergência de preconceitos sociais e estereótipos de uma geração em relação à outra; e dinâmicas relativas à história ou à idiossincrasia local que dificultam este tipo de encontro (Sánchez \& Díaz, 2014).

Assim, atendendo às vantagens, mas também à complexidade de todo o processo, é fundamental que ele assente em bases sólidas, pelo que é pertinente começar a elaboração de um PEI pelo desenho do perfil comunitário. Vários especialistas referem que os PI bem-sucedidos têm em comum partirem das necessidades da comunidade e do contexto (Granville \& Ellis, 1999; Montero \& Gallego, 2002; Bressler, Henkin, \& Adler, 2005; Kuehne, 2005; Newman \& Sánchez, 2007; Springate, Atkinson, \& Martin, 2008; Martin, Springate, \& Atkinson, 2010). O desenho inicial permite compreender, tal como salientam Hatton-Yeo e Watkins (2004), quais são os problemas e as preocupações da comunidade, assim como as oportunidades que a mesma apresenta para o desenvolvimento destes programas, para além de auxiliar a ter discernimento sobre aspetos históricos, a identificar áreas alvo para o desenvolvimento do programa intergeracional e instituições que trabalham na área com o objetivo de perceber quais as oportunidades de estabelecer redes de parcerias e evitar a duplicação do trabalho. Neste âmbito, Newman e Sánchez (2007) afirmam que é importante conectar o programa com a realidade, os recursos, os membros e as necessidades autênticas dessa comunidade. E, no mesmo sentido, Bressler, Henkin, e Adler (2005) asseguram que os programas que se centram na satisfação de necessidades reais e claramente identificadas têm uma maior probabilidade de serem sustentáveis e terem impacto em todos os participantes.

Este trabalho debruça-se, particularmente, sobre os programas educativos intergeracionais (PEI) que são programas que têm como principal função a educação 
(Sáez, 2002), nos quais incluímos os programas de coaprendizagem e programas de serviço e aprendizagem.

Nas linhas que se seguem, e a título de ilustração, apresenta-se o desenho da investigação para de seguida se apresentarem alguns dos resultados e se terminar com a apresentação de possíveis PEI desenhados para a população e comunidade estudadas.

\section{Metodologia e desenho da investigação}

A investigação que aqui apresentamos tem como base um desenho metodológico que combina métodos qualitativos e quantitativos, tendo em conta a abordagem multidimensional que permite considerar os diferentes sectores e fatores individuais, sociais e políticos que subjazem às dinâmicas da vida comunitária (Carmo, 1999; Caffarella \& Daffron, 2013; Pinazo \& Kaplan, 2007). Assim, o plano de análise de necessidades que adotámos desdobrou-se em 3 fases.

$\mathrm{Na}$ primeira fase a investigação centrou-se na análise de necessidades da comunidade: análise documental de dados demográficos estatísticos, localizados em diferentes bases (por exemplo, Instituto Nacional de Estatística (INE), Direção Nacional de Estatística da Educação e Ciência (DGEEC-MEC), etc.); análise de dados das entrevistas a informantes-chave da comunidade e análise de dados das entrevistas focais de grupo a profissionais que trabalham com e junto da população.

Na segunda fase os esforços investigativos centraramse na análise de necessidades dos indivíduos de diferentes gerações que residem na freguesia do Bonfim; análise de dados recolhidos por questionário: Questionário de Necessidades, Interesses e Potencialidade para o Desenvolvimento de Programas Intergeracionais (QNIPDI), aplicado a 385 pessoas com idades compreendidas entre os 15 e os 95 anos, divididos em 3 subamostras etárias, a saber: jovens e a adultos jovens (dos 15 aos 44 anos, $\mathrm{N}=165$ ); adultos de meia-idade (dos 45 aos 64 anos, $\mathrm{N}=110$ ) e adultos idosos dos 65 aos 95 anos, $\mathrm{N}=110$ ). 57\% são mulheres e $43 \%$ homens. E análise de entrevistas semiestruturadas a 20 indivíduos, divididos em 4 subgrupos etários, a saber: jovens (dos 15 aos 24 anos, $\mathrm{N}=5$ ), adultos jovens (dos 25 aos 44 anos, $\mathrm{N}=5$ ), adultos de meia-idade (dos 45 aos 65 anos, $\mathrm{N}=5$ ), e adultos idosos (com 65 ou mais anos, $\mathrm{N}=5$ ); 11 mulheres e 9 homens.

$\mathrm{Na}$ terceira fase, a partir dos resultados obtidos e procedendo à sua integração, elaborou-se um plano exequível de PEI.

Antes de passar a apresentar alguns resultados do estudo, convêm salientar que definimos necessidade como uma discrepância ou diferença entre a situação atual e o que seria desejável (Caffarella \& Daffron, 2013; Witkin e Altschuld, 1995). Mais concretamente, analisam-se as contingências, condições, recursos e potencialidades que é preciso conhecer melhor para ir ao encontro das aspirações mais nobres, que constituam um bem para todos (pessoas e comunidade), não apenas no curto, mas também a médio e longo prazo.

\section{Resultados}

\section{Potencialidades.}

Um património histórico, cultural, económico e social muito rico, elevado número de recursos humanos qualificados, pessoas com níveis de escolaridade muito elevados, boa rede de transportes, rede alargada de instituições, organizações e associações locais, proximidade espacial entre os seus habitantes, sentimento de pertença à cidade do Porto e encontrar-se um conjunto de intereses partilhados por todas as gerações, nomeadamente na dimensão de cuidado e proteção de si e dos outros, dos animais e do ambiente, na dimensão cultura e na dimensão lavores.

\section{Fragilidades, problemas e dificuldades.}

O envelhecimento da população, diminuição da natalidade, perda de habitantes todos os anos, que resulta, sobretudo, da saída "forçada" dos jovens que constituem família para as cidades periféricas devido ao elevado preço do metro quadrado na freguesia, isolamento e solidão social (idosos, desempregados e jovens sem ocupação), aumento de demências e de dificuldades e incapacidades físicas dos adultos idosos, falta de infraestruturas adaptadas à população idosa, a diminuição do número de habitantes, abuso de álcool e drogas, falta de perspetivas e de um plano de vida dos jovens, desemprego, aumento de famílias em risco da pobreza, situações de pobreza extrema, insucesso escolar, abandono escolar, elevado número de famílias monoparentais, violência doméstica, baixa participação na comunidade e sociedade, poucas oportunidades de educação de adultos para adultos de meia-idade e adultos idosos, aglomerados habitacionais como bairros sociais, ilhas e algumas ruas, separação entre as gerações entre outras.

Estes resultados confirmam que a comunidade do Bonfim tem muitas potencialidades para o desenvolvimento de PEI e que se confronta com uma série de dificuldades e fragilidades às quais os PEI podem dar resposta.

\section{Plano de Programas Educativos Intergeracionais}

A nossa sugestão de planificação de PEI é a seguinte:

$1^{\circ}$ Passo - Análise de necessidades, de potencialidades e fragilidades, problemáticas ou dificuldades da comunidade.

$2^{\circ}$ Passo - Decidir qual o programa que queremos colocar em prática e planificar.

Partindo das seguintes perguntas: Quais as necessidades a que quero dar resposta? Quais os objetivos? Quais os interesses das diferentes gerações que posso utilizar para desenvolver o programa? Quem serão os participantes?

Do cruzamento da informação recolhida, interesses e necessidades da população, sistematizamos no Quadro 1 três propostas, a título de exemplo, que nos parecem adequadas à comunidade estudada. 
Quadro 1.

Propostas de PEI para a comunidade do Bonfim

\begin{tabular}{|c|c|c|c|}
\hline \multicolumn{4}{|c|}{$\begin{array}{l}\text { 1 a Proposta: Escolas e centros comunitários } \\
\text { associações, centros de convívio }\end{array}$} \\
\hline \multicolumn{4}{|c|}{$\begin{array}{l}\text { Descrição: Jovens que abandonaram a escola e não } \\
\text { estão a trabalhar e adultos idosos recebem formação } \\
\text { em primeiros socorros, constroem uma ação de } \\
\text { sensibilização para apresentar juntos aos estudantes } \\
\text { das escolas da comunidade do Bonfim. }\end{array}$} \\
\hline $\begin{array}{l}\text { Necessidade } \\
s \\
\text { identificada } \\
S\end{array}$ & $\begin{array}{l}\text { Objetivos } \\
\text { gerais }\end{array}$ & $\begin{array}{c}\text { Interesses } \\
\text { identificados }\end{array}$ & $\begin{array}{l}\text { Partici } \\
\text {-pantes }\end{array}$ \\
\hline $\begin{array}{l}\text { Abandono } \\
\text { escolar. } \\
\text { Jovens sem } \\
\text { perspetivas } \\
\text { e sem um } \\
\text { plano de } \\
\text { vida que se } \\
\text { encontrem } \\
\text { desocupado } \\
\text { s. } \\
\text { Isolamento } \\
\text { de adultos } \\
\text { idosos. } \\
\end{array}$ & $\begin{array}{l}\text { Reduzir o } \\
\text { abandono } \\
\text { escolar dos } \\
\text { jovens. } \\
\text { Promover a } \\
\text { saúde e } \\
\text { prevenir } \\
\text { doenças. } \\
\text { Diminuir o } \\
\text { isolamento } \\
\text { dos idosos. }\end{array}$ & $\begin{array}{l}\text { Aprender a } \\
\text { prestar os } \\
\text { primeiros } \\
\text { socorros. } \\
\text { Ajudar as } \\
\text { outras } \\
\text { pessoas. } \\
\text { Partilhar } \\
\text { conheciment } \\
\text { os com os } \\
\text { outros. }\end{array}$ & $\begin{array}{l}\text { Jovens } \\
\text { que } \\
\text { abandona } \\
\text {-ram a } \\
\text { escola e } \\
\text { não estão } \\
\text { a } \\
\text { trabalhar. } \\
\text { Adultos } \\
\text { idosos. }\end{array}$ \\
\hline \multicolumn{4}{|c|}{$2^{\mathrm{a}}$ Proposta: Escolas e lares de jovens } \\
\hline \multicolumn{4}{|c|}{$\begin{array}{l}\text { Descrição: } \text { Mentoring: adultos idosos ensinam e } \\
\text { ajudam crianças e jovens institucionalizados }\end{array}$} \\
\hline $\begin{array}{l}\text { Necessidade } \\
\text { sidentificad } \\
\text { as }\end{array}$ & $\begin{array}{l}\text { Objetivos } \\
\text { gerais }\end{array}$ & $\begin{array}{c}\text { Interesses } \\
\text { identificados }\end{array}$ & $\begin{array}{c}\text { Partici- } \\
\text { pantes }\end{array}$ \\
\hline $\begin{array}{l}\text { Insucesso } \\
\text { escolar. } \\
\text { Falta de } \\
\text { redes de } \\
\text { apoio social } \\
\text { dos jovens. }\end{array}$ & $\begin{array}{l}\text { Diminuir o } \\
\text { insucesso } \\
\text { escolar dos } \\
\text { jovens. } \\
\text { Alargar a } \\
\text { rede e a } \\
\text { qualidade } \\
\text { do apoio } \\
\text { social a } \\
\text { jovens } \\
\text { institucional } \\
\text { i-zados. }\end{array}$ & $\begin{array}{l}\text { Ensinar e dar } \\
\text { explicações a } \\
\text { crianças e } \\
\text { jovens. } \\
\text { Ajudar as } \\
\text { outras } \\
\text { pessoas. }\end{array}$ & $\begin{array}{l}\text { Crianças } \\
\text { e jovens } \\
\text { institucio } \\
\text { - } \\
\text { nalizados } \\
\text {. Adultos } \\
\text { idosos. }\end{array}$ \\
\hline \multicolumn{4}{|c|}{$\begin{array}{l}3^{\text {a }} \text { Proposta: Centros comunitários, associações, } \\
\text { centros de convívio }\end{array}$} \\
\hline \multicolumn{4}{|c|}{$\begin{array}{l}\text { Descrição: As pessoas envolvidas organizam visitas } \\
\text { guiadas pela freguesia e centro do Porto } \\
\text { fundamentadas na história local e estórias pessoais. }\end{array}$} \\
\hline $\begin{array}{c}\text { Necessidade } \\
\text { s }\end{array}$ & $\begin{array}{l}\text { Objetivos } \\
\text { gerais }\end{array}$ & $\begin{array}{c}\text { Interesses } \\
\text { identificados }\end{array}$ & $\begin{array}{l}\text { Partici- } \\
\text { pantes }\end{array}$ \\
\hline $\begin{array}{l}\text { Desemprego. } \\
\text { Famílias em } \\
\text { risco de } \\
\text { pobreza. } \\
\text { Isolamento } \\
\text { social. }\end{array}$ & $\begin{array}{l}\text { Fomentar } \\
\text { outras formas } \\
\text { de aumentar } \\
\text { rendimentos. } \\
\text { Aumentar o } \\
\text { capital social. } \\
\text { Construir e } \\
\text { aumentar } \\
\text { redes de apoio } \\
\text { social }\end{array}$ & $\begin{array}{l}\text { Ajudar as } \\
\text { outras pessoas. } \\
\text { Partilhar } \\
\text { conhecimentos } \\
\text { com os outros. } \\
\text { Conhecer a } \\
\text { história do } \\
\text { país, cidade e } \\
\text { freguesia. }\end{array}$ & $\begin{array}{l}\text { Pessoas de } \\
\text { todas as } \\
\text { idades } \\
\text { isoladas e } \\
\text { integradas. } \\
\text { Desempre- } \\
\text { gados. }\end{array}$ \\
\hline
\end{tabular}

Planificar, neste passo, as atividades, os conteúdos, a duração do programa e a avaliação, tendo em conta que esta fase da planificação é apenas um projeto que vai ser transformado ao longo da planificação de um PEI que é sempre flexível e deve envolver parceiros e participantes.

$3^{\circ}$ Passo - Reunir organizações relevantes para a estratégia do Programa.

Responder às seguintes perguntas: o que tem a minha organização para o desenvolvimento do PEI? O que é que preciso? Quais os parceiros a procurar?

É importante que se distingam os parceiros colaboradores que se vão envolver e trabalhar no PEI diretamente, e os parceiros que vão cooperar no PEI mas que não serão envolvidos no processo de planificação e implementação (por exemplo, que irão fornecer recursos ou ajudar na divulgação do PEI).

\section{$4^{\circ}$ Passo - Formação do staff.}

Formar em PEI e EI todas as pessoas que fazem parte da minha organização e dos parceiros para que compreendam o programa, os seus objetivos e o seu funcionamento. E formar sobre estereótipos existentes em relação às diferentes gerações.

$5^{\circ}$ Passo - Reunir os parceiros e replanificar o PEI.

Reunir com os parceiros e rever a planificação, solicitando sugestões para melhorar o programa. E definir o papel e os deveres de cada instituição.

$6^{\circ}$ Passo - Recrutamento.

Selecionar participantes que sentem que o PEI dá resposta a uma necessidade pessoal, que têm interesse na atividade e vontade de participar.

$7^{\circ}$ Passo - Desenvolvimento e planificação das atividades com os participantes.

A participação das pessoas na planificação significa reconhecer o direito de escolher, tomar decisões faz com que ninguém se sinta usado ou obrigado, aumentando-se assim o interesse pessoal e o sentimento de pertença ao programa.

$8^{\text {a }}$ Passo - Avaliação.

\section{Conclusão}

O envelhecimento da população é um fenómeno atual que as previsões indicam que sofrerá um agravamento nas próximas décadas (Comissão Europeia, 2014; Eurostat, 2013). Defendemos que este fenómeno deve ser encarado como uma oportunidade e não como um problema. Sendo para isso necessário, um investimento e comprometimento por parte de investigadores, educadores, políticos, organizações e instituições locais, na promoção das relações intergeracionais e na educação intergeracional. Neste trabalho apresentámos o que acreditamos ser uma boa estratégia para atingir este fim - os PEI - e procurámos dar a nossa contribuição, com a ilustração do que consideramos ser a melhor forma de planificar esses programas.

\section{Referencias}

Boström, A-K. (2014). Reflections on intergenerational policy in Europe: The past twenty years and looking into the future. Journal of Intergenerational Relationships, 12(4), 357-367. doi:10.1080/15350770.2014.961828

Bressler, J., Henkin, N., \& Adler, M. (2005). Connecting generations, strengthening communities: A toolkit for intergenerational program planners. 
Philadelphia, PA: Center for Intergenerational Learning, Temple University.

Brink, S. (2017). Learning in later years in the lifelong learning trajectory. Journal of Intergenerational Relationships, 15(1), $14-25$. doi:10.1080/15350770.2017.1260391

Cabanillas, C. (2011). Intergenerational learning as an opportunity to generate new educational models. Journal of Intergenerational Relationships, 9(2), 229 231. doi: 10.1080/15350770.2011.568347

Caffarella, R., \& Daffron, S. (2013). Planning programs for adult learners: A practical guide ( $3^{\mathrm{a}}$ ed.). San Francisco: Jossey-Bass.

Carmo, H. (1999). Desenvolvimento comunitário. Lisboa: Universidade Aberta.

Comissão Europeia (2014), The 2015 Ageing Report. Brussels: European Commission. Acedido em junho de 2017.2 Disponível em: http://ec.europa.eu/economy_finance/publications/eur opean_economy/2015/pdf/ee3_en.pdf

Dorfman, L., Murty, S., Ingram, J., \& Evans, R. (2002). Incorporating intergenerational service-learning into an introductory gerontology course. In M. Mellor \& J. Ivry (Eds.), Advancing gerontological social work education (pp. 219-240). Binghamton, NY: Haworth.

Eurostat (2013). European social statistics, pocketbooks. Luxembourg: Publications Office of the European. Acedido em junho de 2017. Disponível em: http://ec.europa.eu/eurostat/documents/3930297/5968 986/KS-FP-13-001-EN.PDF/

Formosa, M., Krasovec, S., \& Schmidt-Hertha, B. (2014). Conclusion: Policy futures in older adult education and intergenerational learning. In B. Schmidt-Hertha, S. Krasovec \& M. Formosa (Eds.), Learning across generations in Europe (pp. 203-212). Rotterdam: Sense.

Hatton-Yeo, A. (2002). Conference report: Connecting generations: A global perspective. In ICIP International intergenerational conference connecting generations: A global perspective (pp. 1-48). Keele: UNESCO Institute for Education and Beth Johnson Foundation.

Hatton-Yeo, Alan, \& Watkins, Celeste (2004). Intergenerational community development: Practice guide. London: The Beth Johnson Foundation.

Kaplan, M. (2001). Intergenerational programs in schools: Considerations of form and function. International Review of Education, 48(5), 305-334.

Mannion, Greg (2012). Intergenerational education: The significance of reciprocity and place. Journal of Intergenerational Relationships, 10(4), 386-399.

Martin, K., Springate, I., \& Atkinson, M. (2010). Intergenerational practice: Outcomes and effectiveness - LGA Research Report. Slough: NFER.

Montero, I., \& Gallego, A. (2002). Los programas intergeneracionales: Una alternativa socio-educativa para todas las edades. In J. García \& M. Bedmar (Coords.), Hacia la educación intergeneracional (pp.167-177). Madrid: DyKinson.

Newman, Sally, \& Sánchez, Mariano (2007). Los programas intergeneracionales: Concepto, historia y modelos. In Mariano Sanchéz (Dirs.), Programas intergeneracionales: Hacia una sociedad para todas las edades (pp. 34-69). Barcelona: Fundación «la Caixa».

O’Neill, P. (2016). Intergenerational gatherings among the water and willows. In M. Kaplan, L. Thang, M. Sánchez, \& J. Hoffman (Eds.), Intergenerational contact zones - A compendium of applications. University Park, PA: Penn State Extension.

Ohsako, T. (2002). The role of intergenerational programs in promoting lifelong learning for all ages. In C. Mendel-Añonuevo (Ed.), Integrating lifelong learning perspectives (pp. 186- 207). Hamburg: UNESCO Institute for Education.

Pinazo, S., \& Kaplan, M. (2007). The benefits of intergenerational programmes. In M. Sánchez (Dir.), Intergenerational programmes: Towards a society for all ages, (pp. 64-91). Barcelona: The "la caixa" Foundation.

Sáez, J. (2002). Hacia la educación intergeneracional: Concepto y posibilidades. In J. Sáez (Coord.), Pedagogía social y programas intergeneracionales: Educación de personas mayores (pp. 99-112). Málaga: Aljibe.

Sánchez, Mariano, \& Díaz, Pilar (Eds.). (2014). Certificado europeo en aprendizaje intergeneracional: Materialesde formación. Granada: The Beth Johnson Foundation, Association Generations, Högskolan för Lärande och Kommunikation i Jkpg AB, Universidad de Granada \& Dru`stvo za izobrǎzevanje za tretje zivljenjsko obdobje.

Savater, F. (2006). O valor de educar. Lisboa: Publicações Dom Quixote.

Seedsman, T. (2014). The pursuit of continuous improvement in the field of intergenerational relationships: The discipline of the second curve. Journal of Intergenerational Relationships, 12(4), 347356. doi:10.1080/15350770.2014.959442

Simons, L., \& Cleary, B. (2006). The influence of service learning on students' personal and social development. College Teaching, 54(4), 307-319. doi:10.3200/CTCH.54.4.307-319

Springate, I., Atkinson, M., \& Martin, K. (2008). Intergenerational practice: A review of the Literature LGA Research Report. Slough: NFER. Retrieved from

https://www.nfer.ac.uk/publications/LIG01/LIG01.pdf

Teater, B. (2016). Intergenerational programs to promote active ageing: The experiences and perspectives of older adults. Activities, Adaptation \& Ageing, 40(1), 1-19. Retrieved from http://dx.doi.org/10.1080/01924788.2016.1127041

Witkin, B. \& Altschuld, J. (1995). Planning and Conducting Needs Assessments: A Practical Guide. Thousand Oaks, CA, London, New Delhi: Sage Publications.

\section{Agradecimientos}

Co-financiado pela Fundação para a Ciência e Tecnologia. 